\title{
Past the Schwinger limit
}

Science moves forward, at least in part, by pursuing extremes - seeking what's faster, hotter, more stable or more accurate than anything before. We take matter apart into ever smaller pieces, slam particles together at ever higher energies, and manufacture telescopic devices that see ever more deeply into space. In chasing such extremes, we often pass milestones, sometimes almost without noticing.

Seventy years ago, Werner Heisenberg wrote a paper with his student Hans Euler in which they explored the nature of quantum corrections to the classical equations of electrodynamics. They showed that these corrections imply the nonlinear behaviour of the electromagnetic field, much as one finds for Maxwell's equations in a nonlinear medium - for light in an optic fibre, for example. In quantum theory, in effect, the vacuum is also a 'medium', as we now take for granted. Indeed, theorists have shown that the vacuum should participate in fascinating effects ranging from the creation of electron-positron pairs, to photons moving along closed paths, or the self-focusing of beams in free space.
Yet until fairly recently, most of these nonlinear peculiarities expected in electrodynamics have remained theoretical fantasies, owing to our inability to produce the extreme conditions required to see them. But with the rapid and continuing advancement of laser technology, researchers are now getting close. We're set to reach the point - seemingly quite soon - where we can literally bring the vacuum to a boil.

Nonlinear effects associated with the vacuum should be important if the field strength is comparable to $m_{\mathrm{e}} c^{2} / e \lambda_{\mathrm{e}}$, where $\lambda_{\mathrm{e}}$ is the electron Compton wavelength. This is the so-called Schwinger limit, developed by Julian Schwinger in 1951, and works out to be $10^{18}$ $\mathrm{V} \mathrm{m}^{-1}$. This value is surpassed routinely in supernovae and other astrophysical phenomena, but not yet in the lab. At SLAC in the US and DESY in Germany, free electron lasers now being constructed should reach energy densities pushing $10^{29}$ $\mathrm{J} \mathrm{m}^{-3}$, which implies electric fields of about $10^{20} \mathrm{~V} \mathrm{~m}^{-1}$, two orders of magnitude above the Schwinger limit. Even before then, however, the limit may be passed by boosting the capabilities of weaker lasers.

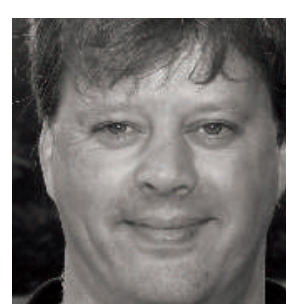

Take the sci-fi-sounding idea of the 'relativistic flying parabolic mirror'. On entering plasma, a powerful laser pulse produces an electric field in its wake, which accelerates electrons - a technique that holds promise for tabletop particle accelerators. But it may also prove useful for boosting the power
HEISENBERG

AND EULER

COULD SEE THIS

MILESTONE ON

THE HORIZON

OF EXTREMES,

YET I DOUBT

THEY THOUGHT

WE'D REACH IT

SO QUICKLY. of laser pulses. Nonlinear effects tend to press the accelerating electrons into dense bunches, and if some of these electrons later meet a counterpropagating laser pulse, they act as a flying mirror - and a relativistic one at that - which should compress the pulse, shift its frequency, and amplify its power. Some teams believe they will be able to use such techniques to blow past the Schwinger limit in the near future.

At that point, we'll be able to see photons scattering off one another and lasers that interact by churning up streams of electrons and positrons. Astrophysics will enter the laboratory in laser-plasma experiments that probe the physics of planetary interiors or the dynamics of supernova shocks. Heisenberg and Euler could see this milestone on the horizon of extremes, yet I doubt they thought we'd reach it so quickly.

Mark Buchanan

\section{Nobel nightmares}

This year's Nobel Prize in physics was awarded to John Mather and George Smoot, for the Cosmic Background Explorer (COBE) observations of the spectrum of and the primordial fluctuations in the cosmic microwave background radiation. This is a well-deserved and long-predicted prize. The COBE discovery changed the way we've done cosmology ever since.

It put aside scepticism that primordial fluctuations would ever be observed, and unleashed a powerful new tool for investigating the Universe. Dozens of experiments have since begun to explore the cosmic microwave background, the latest being the Wilkinson Microwave Anisotropy Probe, which has so far pinned down almost all of the fundamental parameters in cosmology. The probe was named for the late David Wilkinson, a pioneer and leader in the field, who would probably have been the third recipient of the prize with Mather and Smoot. I wish they could leave an empty chair for him on the stage in Stockholm.

At NASA - where COBE was created and housed - a publicrelations officer removed discussion of the Big Bang from their website this year because of the same religious concerns that made school boards censor evolution. I imagine a NASA press release today saying, "We are thrilled with this award to a NASA experiment, but the Big Bang is still only a theory!".

The real tragedy is that this prize underscores the almost

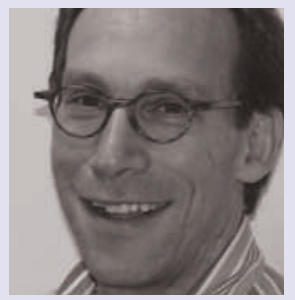

THIS PRIZE UNDERSCORES THE ALMOST COMPLETE DEMISE OF EXCITING SCIENCE AT NASA. complete demise of exciting science at NASA in favour of a white elephant, called the International Space Station, and an expensive race to put humans back on the Moon.

When NASA has done science, it has done so with unmanned missions. The satellites developed to carry on this work - including discovering primordial gravitational waves from the Big Bang - have been delayed or cancelled as NASA shifted $\$ 2$ billion from science into the manned space programme. A nation with the wealth of the US can surely take on the challenge of putting humans into space and still do great science.

Lawrence M. Krauss 